DR GÁBOR MÉHES (Orcid ID : 0000-0003-2503-7451)

Article type : Original Article

\title{
Hypoxia related carbonic anhydrase IX expression is associated with unfavourable response to first-line therapy in classical Hodgkin's lymphoma
}

\author{
Gábor Méhes ${ }^{1}$, Orsolya Matolay ${ }^{1}$, Lívia Beke ${ }^{1}$, Marianna Czenke ${ }^{1}$, Ádám Jóna ${ }^{2}, Z_{\text {Zsófia }}$ \\ Miltényi ${ }^{2}$, Árpád Illés ${ }^{2}$, Judit Bedekovics ${ }^{1}$ \\ ${ }^{1}$ Department of Pathology, Faculty of Medicine, University of Debrecen, Debrecen, Hungary \\ ${ }^{2}$ Hematology Division, Department of Internal Medicine, University of Debrecen, Debrecen,
Hungary
}

Correspondance:

Gábor Méhes MD, PhD

Department of Pathology

Faculty of Medicine, University of Debrecen

Nagyerdei krt. 98.

H-4032 Debrecen, Hungary

E-mail: gabor.mehes@med.unideb.hu

Phone/Fax: +3652255245

Running title: CAIX in classical Hodgkin lymphoma

Conflict of interest statement: no conflict of interest

This article has been accepted for publication and undergone full peer review but has not been through the copyediting, typesetting, pagination and proofreading process, which may lead to differences between this version and the Version of Record. Please cite this article as This article is protected by copyright. All rights reserved. 


\begin{abstract}
Aims: The present study evaluates the impact of hypoxia related carbonic anhydrase IX and XII isoenzyme expression as a basic adaptive mechanism to neutralize intracellular acidosis in classical Hodgkin's lymphoma (cHL).

Methods: 81 primary biopsies and 15 relapsed tissue samples diagnosed with $\mathrm{CHL}$ were analyzed for necrosis, CAIX and CAXII expression and cell proliferation to compare hypoxia related histological and functional data with survival characteristics.

Results: Variable, but highly selective cell membrane CAIX expression could be demonstrated in Hodgkin-Reed-Sternberg (HRS) cells in 39/81 samples (48.1\%) while virtually no staining presented in their microenvironment. In contrast, CAXII expression in HRS-cells could be demonstrated in only $18 / 77$ samples (23.4\%) with significant stromal positivity $(50 / 77,64.9 \%)$. The CAIX+ phenotype was strongly associated with lymphocyte depletion (4/4, 100\%) and nodular sclerosis (29/51, 56.9\%) subtypes. CAIX/Ki-67 dual immunohistochemistry demonstrated suppressed cell proliferation in CAIX+ compared to CAIX-HRS-cells ( $p<0.001) .72$ months progression free survival (PFS) was significantly lower for the CAIX+ group (0.192) compared with the CAIX-group $(0.771)(p<0.001)$ while the overall survival (OS) did not differ ( $p=0.097)$.

Conclusion: Hypoxic stress related adaptation - highlighted by CAIX expression - results in cellular quiescence in HRS-cells potentially contributing to the short term failure of the standard chemotherapy in $\mathrm{CHL}$.
\end{abstract}

Key words: hypoxia, adaptation, cell cycle, resistance, survival, Hodgkin's lymphoma

\title{
Introduction
}

Classical Hodgkin's lymphoma (cHL) is a malignant lymphatic neoplasia of B-cell origin characterized by unique cellular changes including the generation of highly atypical neoplastic proliferation surrounded by a variable extent of reactive elements (1). The mononuclear Hodgkin- and multinucleated Reed-Sternberg cells represent a morphological spectrum of the aborted B-cell maturation also referred as HRS-cells (2). The genomic features and related phenotypic changes of the neoplastic compartment had been largely

This article is protected by copyright. All rights reserved. 
uncovered $(3,4)$. Despite the generally favourable outcome, $\mathrm{CHL}$ is complicated with therapy resistance or disease progression in $25-30 \%$ of the patients (5).

Classical HL is frequently accompanied by variable amounts of focal necrosis and stromal reaction, partially appearing as a consequence of regional perfusion defects especially in the progressive forms of the disease with nodular sclerosis or lymphocyte depletion (syntitial variant) morphology $(6,7)$. Similar to many other malignancies, hypoperfusion and related metabolic disbalance may significantly influence the growth characteristics and the biological responsiveness of neoplastic HRS-cells (8). Hypoxia induces a series of adaptive changes in neoplastic cells the majority of which fall under the control of some key regulators, such as hypoxia inducible factor 1 (HIF-1)(9). The accumulation of HIF-1 $\alpha$ was demonstrated in an $\mathrm{O}_{2}$-tension dependent manner inducing a profound genomic reprogramming with the involvement of several hundreds of genes in both normal and transformed cells (10). One of the key issues of tumor cell survival following hypoxia is the ability to neutralize intracellular acidosis due to enhanced glucose utilization and lactic acid production. The HIF-1 $\alpha$ dependent upregulation of membrane carbonic anhydrase IX and XII (CAIX and CAXII) is an important way to eliminate protons and normalize cytosolic $\mathrm{pH}$ in cancer cells $(11,12)$. CAIX and CAXII are a prominent members of the carbonic anhydrase enzyme family catalyzing bicarbonate production and thus enabling intracellular proton carboxylation and excretion (13). Physiological CAIX and CAXII expression is limited to a small set of normal cell types of epithelial origin associated with special $\mathrm{pH}$ regulatory function, such as the proxymal tubuli of the nephron or the gastrointestinal mucosa (14). However, HIF-1 dependent carbonic anhydrases were repeatedly demonstrated to be abnormally overexpressed in cancer cells. While conventional renal cell cancer and several epithelial cancer types (gastric and lung adenocarcinomas) showed intrinsic CAIX positivity many other neoplastic tissues and especially especially non-epithelial cancers (including glioblastoma) expressed CAIX and/or CAXII in a hypoxia dependent fashion $(15,16,17)$. Based on several studies a strong prognostic significance of CAIX in different types of cancer (e.g. breast, head and neck, stomach, liver, pancreas, colorectal carcinomas) was suggested (18)

Information about CAIX or CAXII in human lymphatic tissues is surprisingly limited to date. Speculations on the potential influence of hypoxia on the biology of $\mathrm{CHL}$ were released earlier based on experimental Hodgkin lymphoma model studies $(8,19)$ and an increased

This article is protected by copyright. All rights reserved. 
CAIX activity could be more specifically measured in CHL samples (20). In agreement with these observations hypoxia related dynamic upregulation of CAIX in cultivated B-cell lineages was reported more recently (21). However, clinical aspects were not addressed so far and currently little is known about the role of adaptive cellular response in the development and outcome of $\mathrm{cHL}$. In the present paper data are provided demonstrating the highly selective expression of CAIX but not CAXII in the neoplastic compartment of $\mathrm{CHL}$ in a significant number of cases, also reflecting regional differences within the same lymphoma tissue. We propose that CAIX related functional changes indicate to a special outfit of the involved neoplastic cells potentially requiring a different anti-cancer strategy.

\section{Materials and Methods}

\section{Sample selection}

Diagnostic samples from 81 patients with classical Hodgkin's lymphoma identified between 1999 and 2015 were reviewed from the archive of the Department of Pathology, University of Debrecen. Ethical approval was provided by the national Scientific Ethical Council (no. $60355-2016 / E K U)$. Histological diagnosis of $\mathrm{CHL}$ was stated according to the current WHO classification (1). The cohort consisted of cases with various subtypes including 51 nodular sclerosis (NS), 20 mixed cellularity (MC), 6 lymphocyte rich (LR), 4 lymphocyte depletion (LD) morphology. Additional (follow up) biopsy material was available in 15 cases of the same cohort.

Clinical data were collected and disease outcome was determined for a follow up period of 72 months after diagnosis. Treatment of the patients followed the actual international recommendations - the standard doxorubicin, bleomycin, vinblastine and dacarbazine (ABVD) combination chemotherapy \pm radiotherapy - with intensification and/or autologous stem cell transplantation in case of failure of response or progression. Overall survival (OS) and progression free survival (PFS) was calculated for each case in months, based on electronic patients records.

\section{Immunohistochemistry}

Representative lymph node biopsy samples were evaluated for morphological signs of focal tissue necrosis following H\&E staining, core needle biopsy samples were were not included. Immunohistochemistry was done on serial sections using the rabbit polyclonal antibody

This article is protected by copyright. All rights reserved. 
clones NB100-417 (Novus Biologicals, Littleton, CO; final dilution 1:2000) for CAIX and PA552608 (Thermo-Fisher Scientific, Rockford, IL) for CAXII. CD30 antigen was demonstrated by the antibody clone Ber-H2 (Dako-Agilent, Glopsrup, Danemark) and EBV-related LMP-1 protein by the antibody clone CS1-4 (Dako-Agilent). Specific antibody binding was highlighted using the EnVision Flex HRP/DAB+ chromogen detection system (Dako-Agilent) following antigen retrieval at pH9.0 as usual. Reactive/uninvolved lymph node samples were used as negative controls which never presented with any CAIX or CAXII specific signals. Kidney tissue samples with renal cell cancer involvement were used as positive control staining where normal and neoplastic cells of tubular origin showed strong membrane positivity for CAIX and XII as expected.

Cell proliferation in individual CAIX+ cells was rated following dual IHC reaction for CAIX and $\mathrm{Ki}-67$ in all cases showing a positive reaction as follows. The nuclear Ki-67 protein was specifically labeled with the antibody clone Mib1 (Dako-Agilent; dilution 1:200) and detected by the EnVision Flex HRP/DAB+ (brown color) procedure. This was followed by a second incubation with the CAIX binding primary antibody (see above) that was detected by the Flex HRP system using the violet VIP chromogen (Vector Laboratories, Burlingame, CA) as a substrate. This setting clearly differentiated the Ki67 related brown nuclear staining and the CAIX specific violet cell membrane staining within the same cells by light microscopy. Methyl-green solution (Vector Laboratories) was used to counterstain unlabeled cell nuclei for tissue orientation.

\section{Data evaluation}

CAIX positive slides were digitalized using the Pannoramic digital slide scanner device (3DHistech, Budapest, Hungary) and were further analyzed by the Pannoramic Viewer software on the screen. HRS cells with and without membrane bound CAIX or CAXII expression were counted based on the cell size and morphology. Slides with more than 10 immunostained HRS cells were defined as unambigous positive. Non-HRS positivity of the microenvironment was separately documented, however, quantification was not enabled. For the evaluation of the dual-IHC stained samples up to 300 HRS cells (depending on their total mass) with or without CAIX positivity were considered from different areas of the same slide. Sections containing less than 30 representative CAIX+ cells were not considered. Nuclear Ki-67-positivity within the CAIX-positive and negative HRS-cell fraction was

This article is protected by copyright. All rights reserved. 
determined and the relative frequency of $\mathrm{Ki}-67$ expressing cells for both groups was calculated.

Statistical analysis including the Fisher's exact test and the Kaplan-Mayer survival analysis was performed using the IBM SPSS Statistics Version 24 software (IBM Analytics).

\section{Results}

CAIX and CAXII immunostaining pattern in $\mathrm{CHL}$

Serial sections with definitive $\mathrm{CHL}$ involvement were evaluated for both CAIX and CAXII immunopositivity. We observed a limited, but highly selective positive membrane reaction on individual cells with typical HRS morphology when CAIX specific antibody was applied (Figure 1A). In contrast, CAXII immunostaining resulted in more indefinite positive areas consisted mostly of stromal and immune cell types sometimes also including HRS-cells. (Figure 1B). Although both CAIX and CAXII labeling reflected focal areas of the neoplastic proliferation, only CAIX proved to be selective to highlight clusters of HRS-cells within masses of non-labeling atypical cells (Figure $1 \mathrm{C}$ and D). On the other hand, the distinction between neoplastic HRS cells and the reactive infiltrate of the microenvironment seemed to be impossible for most of the CAXII immunostained cases.

Initial lymph node samples from 81 patients (male to female ratio was 40:41) with the histological diagnosis of $\mathrm{CHL}$ were evaluated for characteristics of CAIX enzyme expression. CAIX immunopositivity presented with a clear membrane reaction in $39 / 81$ samples $(48.1 \%)$ (Table 1). CAIX+ HRS cells were generally located in small dyscohesive clusters but isolated single cells could also be frequently found. CAIX positivity ranged from scattered isolated HRS-cells through several small clusters to large sheats of cells with atypical morphology and almost uniform strong immunopositivity (up to $80.0 \%$ ). In contrast, cells not equivalent with HRS-morphology were generally not marked the same way. Small foci of stromal cells surrounding strongly positive HRS cells with a weak and indefinite staining were only occasionally present (2/81 samples, $2.5 \%)$.

The labelling pattern was different for CAXII where only 18/77 evaluated samples (23.4\%) displayed distinct membrane HRS-cell positivity. In addition, stromal and immune cell reaction could be demonstrated in 50/77 cases (64.9\%) which was associated with the CAXII+ neoplastic compartment but more frequently occured independently due to activity of fibrosis and chronic inflammatory infiltrate.

This article is protected by copyright. All rights reserved. 


\section{CAIX, CAXII and tissue necrosis in CHL subtypes}

As expected, we found a clear morphological relation between the appearance of tissue necrosis primarily stated in $\mathrm{HE}$-stained slides and the CAIX expressing neoplastic compartment (Table 1.). Classical necrotic foci presented with a central amorphous mass surrounded by several layers of CAIX-positive HRS cells demonstrated by immunohistochemistry (Figure 2). This repeatedly indicated a direct relation between sublethal hypoxic damage and CAIX upregulation in the marginal zone lining necrotic foci. CAIX+ was present in the great majority $(18 / 21)$ of the samples featured with necrosis (85.7\%). However, CAIX expression frequently occured in the absence of clear necrotic morphology. While unambigous signs of necrosis could be identified in only $21 / 81$ (25.9\%), CAIX positivity appeared in $39 / 81(48.1 \%)$ of the samples. CAIX positivity was followed by necrosis in only $18 / 39$ samples $(46.2 \%)(p<0.0001)$ (Table 1.$)$.

CAIX expression and necrosis were both found to be strongly dependent on the histological subtype of Hodgkin's lymphoma (Table 2). All four LD cases (100.0\%) featured with massive geographical necrosis and large areas of CAIX+ neoplastic cells. While 29/51 (56.9\%) of the NS cases presented with variable amounts of CAIX expression only $5 / 20(25.0 \%)$ of the MC subgroup and a single case of the LR subgroup $(1 / 6,16.7 \%)$ showed CAIX expression. Differences between histological subentities were not that striking when CAXII expression was tested, except the strong correlation of CAXII with the LD subgroup $(4 / 4,100 \%$ ) (Table 2.).

\section{Cell proliferation and CAIX expression in HRS-cells}

In order to evaluate the general functional status of CAIX-expressing HRS cells we applied the Ki67 cell proliferation marker in a double IHC setting. HRS-cells were identified on digital slides based on their morphology following methyl-green counterstaining. Cells with clear membrane IHC staining in violet (VIP) were declared as CAIX+ while brown nuclear staining (DAB) referred to Ki67 positivity (Figure 3). Following the double IHC evaluation in 26 samples applicable (more than 30 HRS-cell available for the analysis) we stated that the overall Ki67 positivity (reflecting proliferation capacity) in CAIX+ cells was significantly lower, than that of the CAIX negative tumor cell compartment. The range of Ki67 positivity extended between $0.3-51.4 \%$ (mean $29.72 \pm 13.07$ ) for the CAIX+ and between $51.2-92.0 \%$ (mean $69.38 \pm 10.79$ ) for the CAIX negative HRS-cell population ( $p<0.001$ ) (Figure 4.).

This article is protected by copyright. All rights reserved. 


\section{CAIX and CAXII status in rebiopsy samples}

15 out of the 81 cases included in our evaluation had accessible rebiopsy samples taken due to therapy failure or recurrance. $13 / 15$ cases belonged to the CAIX expressing $\mathrm{CHLs}$ (86.6\%). CAIX immunostaining in these samples presented a special dinamics and was slightly different from the initial status. Five rebiopsy samples (33.3\%) changed to negative during the course of the treatment while the rest of CAIX+ cases also showed a reduced frequency of CAIX positive cells in the follow up samples. On the other hand, none of the negative samples converted to positive and significant increase in CAIX expression was also never detected in the follow-up series.

CAXII staining revealed similar results comparing primary and follow-up lymph node biopsies. HRS-cell positivity remained unchanged in 10/15 rebiopsy samples (66.6\%) while turned to negative following initial CAXII positivity in $5 / 15$ samples (33.3\%).

\section{Clinical impact of CAIX and CAXII expression in CHL}

Clinical data for 72 months total time period were collected and evaluated for the influence of necrosis as well as CAIX and CAXII immunopositivity observed in the primary biopsy sample on general survival for all available cases. The longest survival measured was 138 months from the initial diagnosis. The overall survival (OS) rate was 0.823 and the progression free survival (PFS) was 0.504 for the total study population $(n=81)$, both results reflecting the current clinical standards.

Accordidng to the Kaplan-Meier curves and the Mantel-Cox statistical analysis the presence or absence of necrosis in $\mathrm{cHL}$ did not influence the overall survival ( 0.812 vs $0.833, p=0.469$ ) and despite a visible mild difference in the PFS, cases featuring tissue necrosis did not perform statistically worse compared to the non-necrotic cases ( 0.19 vs $0.579, p=0.146$, not significant) (Figure $5 \mathrm{~A}-\mathrm{B})$. The centralized Wilcoxon (Breslow) test considering the outcome and the duration of the follow up revealed the scores 0.724 and 0.464 , respectively. Interestingly, a more striking difference was found in relation to CAIX expression (Figure 5CD). While the OS differed only minimally between the CAIX-negative and CAIX-positive groups ( 0.934 vs $0.699, p=0.097$, statistically not significant), PFS proved to be highly different depending on the CAIX status. Our comparison reflected a statistically highly significant longer cumulative progression free survival in the CAIX- $(0.771)$ compared to the CAIX+ cases (0.19) $(p<0.001)$ (Figure $5 C-D)$. The Breslow-scores were 2.032 for OS and 12.162 
for PFS. In contrast, OS and PFS did not significantly differ between the CAXII negative and positive lymphomas ( $p=0.301$ and $p=0.524$, respectively) in the 77 cases evaluated (Breslowscores were 1.977 and 0.649 , respectively)(Figure $5 E-F$ ).

The positive predictive value (PPV) for an initial treatment failure proved to be 0.76 in case of CAIX positivity while the negative predictive value (NPV) was 0.66 for lack of progression in the absence of CAIX expression determined in the initial biopsy sample $(p<0.003)$. As expected PPV and NPV for the CAXII was generally weak (statistically not different, no predictive value).

\section{Discussion}

The dynamic overexpression of carbonic anhydrases seems to be an important adaptive feature due to hypoperfusion related tissue hypoxia in selected cancers. The specific role of cell membrane bound CAIX and CAXII to neutralize intracellular acidosis in neoplastic cells was established in in vitro and in vivo models including knock-down animal model experiments (22). CAIX has been investigated histologically in a series of cancers and was found to be associated with aggressive phenotype and/or unfavourable prognosis $(18,23)$. Considerably less information is available for CAXII expression at the histological level.

The potential role of hypoxia in the evolution and progression of $\mathrm{cHL}$ was not in the focus of extended clinical or molecular investigations. Hypoxia related pathways were not reported to be generally activated in HRS cells by gene expression studies (4) or epigenetic profiling $(24,25)$.

Here we demonstrated, that further to hypoxic necrosis, an even more significant fraction of classical Hodgkin's lymphoma is characterized by focal expression of CAIX and CAXII. CAIX IHC showed a strong membrane reaction that proved to be virtually selective for HRS cells with only minimal and indefinite IHC staining in bystanding cells in, usually in close proximity with the strong HRS-cell reaction. Activated large mononuclear tumor cells occasionally seen as CAIX positive were interpreted as "precursor HRS-cells" sharing the pathobiology of the classical cell type. In contrast, CAXII IHC labelled a series of additional cell types further to the neoplastic compartment. In our interpretation the CAXII labelling pattern was consistent with the topography of hypoxic stress generally affecting the complex tissue composition of

This article is protected by copyright. All rights reserved. 
$\mathrm{cHL}$, including inflammatory and regenerative components. Thus, CAXII labeling did not appear to selectively reflect functional changes at the individual cancer cell level and seemed to be of limited utility to present and interpret the extent of hypoxic adaptation in histological conditions.

In contrast to CAXII the association between CAIX expression and spontaneous necrosis could be clearly established by histology in untreated primary $\mathrm{CHL}$ biopsies. HRS cells with CAIX positivity apparently formed a well defined compartment surrounding morphologically recognizable necrotic foci. Moreover, CAIX expressing HRS-cells could be observed in the great majority (85.7\%) of the samples presenting with necrosis by conventional H\&E staining, indicating to a close relation between hypoxic damage and CAIX expression. In reverse, more than half of the samples with CAIX+ compartment did not present with morphological signs of necrosis. According to this observation focal adaptive changes induced by non-lethal hypoxic stress may result in the selective upregulation of CAIX in HRScells and adaptive CAIX expression generally occurs prior massive cell demage in HRS-cells. On the contrary, expression of isoenzyme CAXII - supposed to be regulated by the hypoxia pathway in a similar way - could be demonstrated in neoplastic HRS-cells in the minority (23.4\%) of the evaluated cases together with the labeling of further non-neoplastic cell types (fibroblasts, histiocytes and other immune cells) associated with tissue reparation.

Despite the limited number of cases reflecting different histological subgroups in our cohort we found basic differences regarding hypoxia related features. Frequent occurrance of necrosis and even more frequent CAIX expression in LD and in NS indicate to general adaptive and regressive capabilities in these subtypes (in $100.0 \%$ and $56.9 \%$ of the cases, respectively). Interestingly, all four cases of lymphocyte depleted $\mathrm{cHL}$ presented with areas of necrosis and strong membrane CAIX expression as well as CAXII in up to $80 \%$ of the HRScells in each sample. The LD form presents in the form of a sarcoma like solid tumor with the most unfavourable behaviour among $\mathrm{CHL}$ subtypes. Indeed, three out of the four patients with LD CHL and CAIX/CAXII expression died of disease progression within 14 months after diagnosis. In contrast, the general lack of necrosis and the low frequency CA expression in $\mathrm{LR}$ and $\mathrm{MC}$ subtypes of $\mathrm{CHL}$ suggest that hypoperfusion and hypoxia-related reprogramming is less relevant in the course of these histological forms.

This article is protected by copyright. All rights reserved. 
Importantly, cancer cells suffering from hypoxia and acidosis tend to aquire regressive features also shared by cancer stem cells $(7,26,27)$. The functional analysis of the tumor cell compartment could be done in the present study using a CAIX/Ki-67 double IHC approach. The Ki-67 nuclear protein is a key factor of cell cycle progression $(28,29)$, which can be demonstrated with high affinity by the antibody clone Mib-1 (30). The Ki-67 labeling index is widely used to estimate the proliferative capacity and as such it is an established prognostic factor in different malignant conditions $(31,32,33)$ including lymphoid neoplasias (34). Earlier data reported a generally high cell proliferation activity in HRS-cells with over $90 \%$ cell cycle activity (35) and Ki-67 indices between $57 \%$ and $80 \%(36,37)$. Detailed analysis by Tzankov et al (35) suggested profound cell cycle regulatory defects behind these high overall ratios. CAIX/Ki67 double immunohistochemistry enabled us to differentiate and demonstrate adaptation related cell cycle activity in HRS-cells for the first time. Unfortunately, a dual IHC setting for CAXII/Ki67 failed due to the high number of CAXII+ bystanding cells and unsatisfactory identification of the cell composition required for the analysis. The cell proliferation of CAIX expressing neoplastic cells proved to be strongly repressesed (mean $29.72 \pm 13.07$ ) compared to the CAIX negative compartment (mean $69.38 \pm 10.79$ ) of the same biopsy sample. Despite the generally high proliferation rate, CAIX-positivity was associated with suppressed cell cycle activity in all evaluated samples, irrespective of the histological subtype. Cellular quiescence in the positive HRS-cell compartment is in good agreement with the limited actual energy supply of the immediate tissue environment. This observation further supports the beneficial effect of CAIX expression in hypoxia-related adaptation.

Tissue perfusion is generally more likely to be challanged in aggressive forms of $\mathrm{CHL}$. The functional changes due to hypoxic adaptation therefore may have major clinical impact on progression and therapeutic response in $\mathrm{cHL}$. As a very first attempt we evaluated the clinical course related to tissue necrosis and adaptation driven CAIX and CAXII upregulation in $81 \mathrm{cHL}$ cases. While necrosis had only little effect $(p=0.146)$ and CAXII did not show any influence $(p=0.524)$ on therapy response (PFS), cases with CAIX expression had a highly distinct $(p<0.001)$ unfavourable response to the frontline standard therapy. This effect remained still minimally visible when the overall survival rates were compared $(p=0.097)$. Thus, CAIX expression in $\mathrm{CHL}$ could be associated with the failure of the initial treatment

This article is protected by copyright. All rights reserved. 
according to the standard therapy (ABVD)even in a univariate setting. Both the positive predictive value (PPV) and the negative predictive value (NPV) of CAIX expression indicated to the strongly elevated likelyhood of failure/progression after the initial chemotherapy (76.2 and $65.9 \%$, respectively).

Based on these first observations hypoxia related adaptive mechanisms seem to have an important role in the complex biology of $\mathrm{CHL}$. Especially CAIX was found promising to selectively highlight hypoxic cellular demage in the neoplastic compartment. CAIX immunohistochemistry of the primary biopsy specimen may help to predict initial therapy response and to select high-risk patients right at the diagnosis of classical Hodgkin lymphoma.

\section{Aknowledgements}

Gábor Méhes desigend the study, performed histological evaluations and wrote the manuscript

Orsolya Matolay collected and managed tissue samples, performed histological evaluations, collected data and participated in statistical evaluation

Livia Beke performed histological work up and immunohistochemical stainings

Marianna Czenke did data management, statistics and graphical work

Ádám Jóna collected clinical data and performed statistical evaluations

Zsofia Miltenyi collected clinical data and performed statistical evaluations

Árpád Illés collected clinical and laboratory data and participated in the preparation of the manuscript

Judit Bedekovics performed histological evaluations, participated in sample collection and management, contributed to the written and graphical setup and corrections of the manuscript

This article is protected by copyright. All rights reserved. 


\section{References}

1. Swerdlow SH, Campo E, Pileri SA, et al. The 2016 revision of the World Health Organization classification of lymphoid neoplasms. Blood 2016; 127(20): 2375-2390.

2. Lee IS, Kim SH, Song HG, Park SH. The molecular basis for the generation of Hodgkin and Reed-Sternberg cells in Hodgkins lymphoma. Int J Hematol 2003; 77(4):330-335.

3. Kanzler H, Küppers R, Hansmann ML, Rajewsky K. Hodgkin and Reed-Sternberg cells in Hodgkin's disease represent the outgrowth of a dominant tumor clone derived from (crippled) germinal center B cells. J Exp Med 1996; 184(4):1495-1505.

4. Tiacci E, Döring C, Brune V, et al. Analyzing primary Hodgkin and Reed-Sternberg cells to capture the molecular and cellular pathogenesis of classical Hodgkin lymphoma. Blood 2012; 120(23):4609-4620.

5. Canellos GP, Rosenberg SA, Friedberg JW, Lister TA, Devita VT. Treatment of Hodgkin lymphoma: a 50 years perspective. J Clin Oncol 2014; 32(3):163-168.

6. Lukes RJ, Butler JJ, Hicks EB. Natural history of Hodgkin's disease as related to its pathologic picture. Cancer 1966; 19:317-344.

7. Colby TV, Hoppe RT, Warnke RA. Hodgkin's disease: a clinicopathologic study of 659 cases. Cancer 1982; 49:1848-1858.

8. Wein F, Otto T, Lambertz P, Fandrey J, Hansmann ML, Küppers L. Potential role of hypoxia in early stages of Hodgkin lymphoma pathogenesis. Haematologica 2015; 100(10):1320-1326.

9. Mimeault M, Batra SK. Hypoxia inducing factors as master regulators of stemness properties and altered metablism of cancer- and metastasis initiating cells. J Cell Mol Med 2013; 17(1):30-54.

This article is protected by copyright. All rights reserved. 
10. Cummins EP, Taylor CT. Hypoxia-responsive transcription factors. Pflugers Arch 2005; 450(6):363-371.

11. Chiche J, Ilc K, Laferrière J, Trottier E, et al. Hypoxia-Inducible Carbonic Anhydrase IX and XII Promote Tumor Cell Growth by Counteracting Acidosis through the Regulation of the Intracellular pH. Cancer Res 2009; 69(1):358-368.

12. Pastorek J, Pastorekova S. Hypoxia induced carbonic anhydrase IX as a target for cancer therapy: from biology to clinical use. Semin Cancer Biol 2015; 31:52-64.

13. Sedlakova O, Svastova E, Takacova M, Kopacek J, Pastorek J, Pastorekova S. Carbonic anhydrase IX, a hypoxia induced catalytic component of the $\mathrm{pH}$ regulating machinery in tumors. Front Physiol 2014; doi: 10.3389/fphys.2013.00400.

14. Liao SY, Lerman MI, Stanbridge EJ. Expression of transmembrane carbonic anhydrases, CAIX and CAXII, in human development. BMC Dev Biol 2009; doi: 10.1186/1471-213X-9-22.

15. Proescholdt MA, Merrill MJ, Stoerr EM, Lohmeier A, Pohl F, Brawanski A. Function of carbonic anhydrase in glioblastoma multiforme. Neuro Oncol 2012; 14(11):13571366.

16. Klimowicz AC, Bose P, Petrillo SK, Magliocco AM, Dort JC, Brockton NT. The prognostic impact of a combined carbonic anhydrase IX and Ki67 signature in oral squamous carcinoma. Br J Cancer 2013; 109:1859-1866.

17. Huang WJ, Jeng YM, Lai HS, et al. Expression of hypoxic marker carbonic anhydrase IX predicts poor prognosis in resectable hepatocellular carcinoma. PlosOne 2015; 10(3):e0119181.

This article is protected by copyright. All rights reserved. 
18. van Kuijk SJ, Yaromina A, Houben R, Niemans R, Lambin P, Dubois LJ. Prognostic significance of carbonic anhydrase IX in cancer patients: a meta analysis. Front Oncol 2016; 29 (6):69.

19. Kewitz S, Kurch L, Volkmer I, Stagege MS. Stimulation of the hypoxia pathway modulates chemotherapy resistance in Hodgkin's lymphoma cells. Tumor Biol 2016; 37:8229-8237.

20. Stewart $M$, Talks $K$, Leek $R$, et al. Expression of angiogenic factors and hypoxia inducible factors HIF1, HIF2 and CAIX in non-Hodgkin's lymphoma. Histopathology $2002 ; 40: 253-260$.

21. Chen LQ, Howison CM, Spier C, et al. Assessment of carbonic anhydrase IX expression and extracellular pH in B-cell lymphoma cell line models. Leuk Lymphoma 2015; 56(5):1432-1439.

22. Spugnini EP, Sonveaux $P$, Stock $C$, et al. Proton chanels and exchangers in cancer. BBA 2015; 1848: 2715-2726.

23. Ilardi G, Zambrano N, Merolla F, et al. Histopathological determinants of tumor resistance: a special look to the immunohistochemical expression of carbonic anhydrase IX in human cancers. Curr Medicinal Chem 2014; 21:1569-1582.

24. Doerr JR, Malone CS, Fike FM, et al. Patterned CpG methylation of silenced B-cell gene promoters in classical Hodgkin lymphoma derived and primary effusion lymphoma cell lines. J Mol Biol 2005; 350(4):631-640.

25. Ushmorov A, Leithauser F, Sakk O, et al. Epigenetic processes play a major role in Bcell specific gene silencing in classical Hodgkin lymphoma. Blood 2006; 107(6):24932500 .

This article is protected by copyright. All rights reserved. 
26. Jiang J, Tang YL, Liang XH. EMT: a new vision of hypoxia promoting cancer progression. Cancer Biol Ther 2011; 11:714-723.

27. Foster JG, Wong SC, Sharp TV. The hypoxic tumor microenvironment: driving the tumorigenesis of non-small-cell lung cancer. Future Oncol 2014; 10:2659-2674.

28. Wu L, Timmers C, Maiti B, et al. The E2F1-3 transcription factors are essential for cellular proliferation. Nature 2001; 414(6862):457-462.

29. Spyratos F, Ferrero-Pous M, Trassard M, et al. Correlation between MIB-1 and other proliferation markers: clinical implications of the MIB-1 cutoff value. Cancer 2002; 94(8):2151-2159.

30. Schlüter C, Duchrow M, Wohlenberg C, et al. The cell proliferation-associated antigen of antibody Ki-67: a very large, ubiquitous nuclear protein with numerous repeated elements, representing a new kind of cell cycle-maintaining proteins. J Cell Biol 1993; 123(3):513-522.

31. Kjellman P, Wallin G, Hoog A, Auer G, Larsson C, Zedenius J. MIB-1 index in thyroid tumors: a predictor of the clinical course in papillary thyroid carcinoma. Thyroid 2003; 13(4):371-380.

32. Petrelli F, Viale G, Cabiddu M, Barni S. Prognostic value of different cut-off levels of Ki-67 in breast cancer: a systematic review and meta-analysis of 64,196 patients. Breast Cancer Res Treat 2015; 153(3):477-491.

33. Tian Y, Ma Z, Chen Z, et al. Clinicopathological and prognostic value of Ki-67 expression in bladder cancer: a systematic review and meta-analysis. PLoS One 2016 11(7):e0158891.

This article is protected by copyright. All rights reserved. 
34. Broyde A, Boycov O, Strenov Y, Okon E, Shpilberg O, Bairey O. Role and prognostic significance of the Ki-67 index in non-Hodgkin's lymphoma. Am J Hematol 2009; $84(6): 338-343$

35. Tzankov A, Zimpfer A, Went $P$, et al. Aberrant expression of cell cycle regulators in Hodgkin and Reed-Sternberg cells of classical Hodgkin's lymphoma. Mod Pathol $2005 ; 18(1): 90-96$

36. Aktaş S, Kargi A, Olgun N, Diniz G, Erbay A, Vergin C. Prognostic significance of cell proliferation and apoptosis-regulating proteins in Epstein-Barr virus positive and negative pediatric Hodgkin lymphoma. Lymphat Res Biol 2007; 5(3):175-182.

37. Tiemann $\mathrm{M}$, Claviez $\mathrm{A}$, Lüders $\mathrm{H}$, et al. Proliferation characteristics in pediatric Hodgkin's lymphoma point to a cell cycle arrest in the $\mathrm{G}(1)$ phase. Mod Pathol 2005; 18(11):1440-1447.

\section{Table and Figure legends}

Table 1. Expression of CAIX and CAXII in HRS-cells in relation to tissue necrosis determined by histology in $\mathrm{CHL}$ lymph node biopsies at diagnosis $(n=81)$. The statistically significant positive association between CAIX-positivity and necrotic foci was established by Fisher's exact test ( $p<0.001$, asterix). No correlation between CAXII-expression and necroses could be stated $(p=0.35)$

Table 2. Occurrance of necrosis, CAIX+ and CAXII+ HRS-cells according to histological subtypes in $\mathrm{CHL}$. CAIX-expression was most frequently observed in LD and NS subtypes. All samples of the LD group featured both CAIX+ and CAXII+ HRS-cells (4/4, 100\%), this difference was statistically significant compared to any of the other histological subgroups ( $p<0.001$, independent-samples t-test). (NS, nodular sclerosis; MC, mixed cellularity; LR lymphocyte-rich; LD, lymphocyte depleted)

This article is protected by copyright. All rights reserved. 
Figure 1. Membrane expression of CAIX (A) and CAXII (B) in HRS cells in classical Hodgkins lymphoma. While CAIX immunostaining proved to be virtually selective for the HRS cell morphology spectrum, CAXII positivity was frequently observed in immune cells including histiocytes and stromal fibroblasts ( $\times 40$ magnification, scale bar represents $50 \mu \mathrm{m}$ ).

Figure 2. Double immunohistochemistry highlighting HRS-cells by CD30 (DAB-chromogen, brown) and the CAIX specific staining (VIP-chromogen, violet) in different regions of a representative samples. Scattered CD30+ cells are presented lacking CAIX-positivity in panel $A$ (arrowheads). A cluster of CD30+/CAIX+double immunopositive HRS-cells is indicated by arrows, surrounded by CD30+ HRS-cells lacking CAIX expression (arrowheads) in panel B (both $\times 20$ magnification, scale bar $100 \mu \mathrm{m}$ ). CD30+/CAIX+ double positive cells (arrows) typically arranged as a well defined zone demarcating a focal necrotic core (panel $\mathrm{C})(\times 10$ magnification, scale bar $200 \mu \mathrm{m}$ ).

Figure 3. Double immunohistochemistry highlighting nuclear Ki-67 staining (DAB-brown) and CAIX specific staining (VIP-violet) from two representative sample fields ( $A$ and $B$ ). Note the missing nuclear staining in CAIX+ large HRS cells (arrows) compared to the intense Ki-67 labeling in some of the surrounding large cells lacking CAIX staining (arrowheads) (x100 magnification)

Figure 4. Ki-67 labeling index determined separately in CAIX+ and CAIX-HRS cell compartments within the same untreated $\mathrm{CHL}$ biopsy samples featuring CAIX expressing clusters and applicable for dual IHC analysis $(n=26)$. Ki67 labeling index proved to be significantly lower in CAIX+ HRS-cells $(p<0.001)$ indicating to adaptation related cell-cycle arrest.

This article is protected by copyright. All rights reserved. 
Figure 5. Kaplan-Meier curves reflecting 72 months overall survival (left column) and progression free survival (right column) related to the presence of necrosis ( $A$ and $B$ ), CAIX ( $C$ and $D$ ) as well as CAXII expression ( $E$ and F) in classical Hodgkin lymphoma. Statistically highly significant difference in PFS was calculated in favour of the CAIX- (0.771) compared to $C A I X+(0.19)$ group $(p<0.001)(D)$.

Table 1. Expression of CAIX and CAXII in HRS-cells in relation to tissue necrosis determined by histology in $\mathrm{CHL}$ lymph node biopsies at diagnosis $(n=81)$. The statistically significant exact test ( $p<0.001$, asterix). No correlation and necrotic foci was established by Fisher's stated $(p=0.35)$

\begin{tabular}{|c|c|c|c|c|}
\hline & & \multicolumn{3}{|c|}{ necrosis (\%) } \\
\hline & & pos & neg & total (\%) \\
\hline \multirow{3}{*}{$\begin{array}{l}\text { CAIX }^{+} \\
\text {HRS (\%) }\end{array}$} & & $18(22.2)^{*}$ & 21 (25.9) & $39(48.1)$ \\
\hline & neg & $3(3.7)$ & 39 (48.1) & 42 (51.9) \\
\hline & total & 21 (25.9) & $60(74.1)$ & 81 (100.0) \\
\hline \multirow{3}{*}{$\begin{array}{l}\text { CAXII }^{+} \\
\text {HRS (\%) }\end{array}$} & & 9 (11.7) & $9(11.7)$ & $18(23.4)$ \\
\hline & neg & $12(15.6)$ & 47 (61.0) & 59 (76.6) \\
\hline & total & 21 (27.3) & $56(72.7)$ & 77 (100.0) \\
\hline
\end{tabular}

This article is protected by copyright. All rights reserved. 
Table 2. Occurrance of necrosis, CAIX+ and CAXII+ HRS-cells according to histological
subtypes in CHL. CAIX-expression was most frequenty subtypes in CHL. CAIX-expression was most frequently observed in LD and NS subtypes. All difference was statistically significant cAIX+ and CAXII+ HRS-cells $(4 / 4,100 \%)$, this ( $p<0.001$, independent-samples t-test). (NS, nodu any of the other histological subgroups lymphocyte-rich; LD, lymphocyte depleted)

\begin{tabular}{llll}
\hline & necrosis (\%) & CAIX HRS (\%) & CAXII HRS (\%) \\
\hline \hline NS & $14 / 51(27.5)$ & $29 / 51(56.9)$ & $10 / 49(20.4)$ \\
MC & $3 / 20(15.0)$ & $5 / 20(25.0)$ & $3 / 18(16.7)$ \\
LR & $1 / 6(16.7)$ & $1 / 6(16.7)$ & $1 / 6(16.7)$ \\
LD & $3 / 4(75.0)$ & $4 / 4(100.0)^{*}$ & $4 / 4(100.0)^{*}$ \\
\hline total & $21 / 81(25.9)$ & $39(48.1)$ & $18 / 77(23.4)$
\end{tabular}

This article is protected by copyright. All rights reserved. 
Figure 1. Membrane expression of CAIX (A) and CAXII (B) in HRS cells in classical Hodgkins lymphoma. While CAIX immunostaining proved to be virtually selective for the HRS cell morphology spectrum, CAXII positivity was frequently observed in immune cells including histiocytes and stromal fibroblasts ( $\times 40$ magnification, scale bar represents $50 \mu \mathrm{m}$ ).
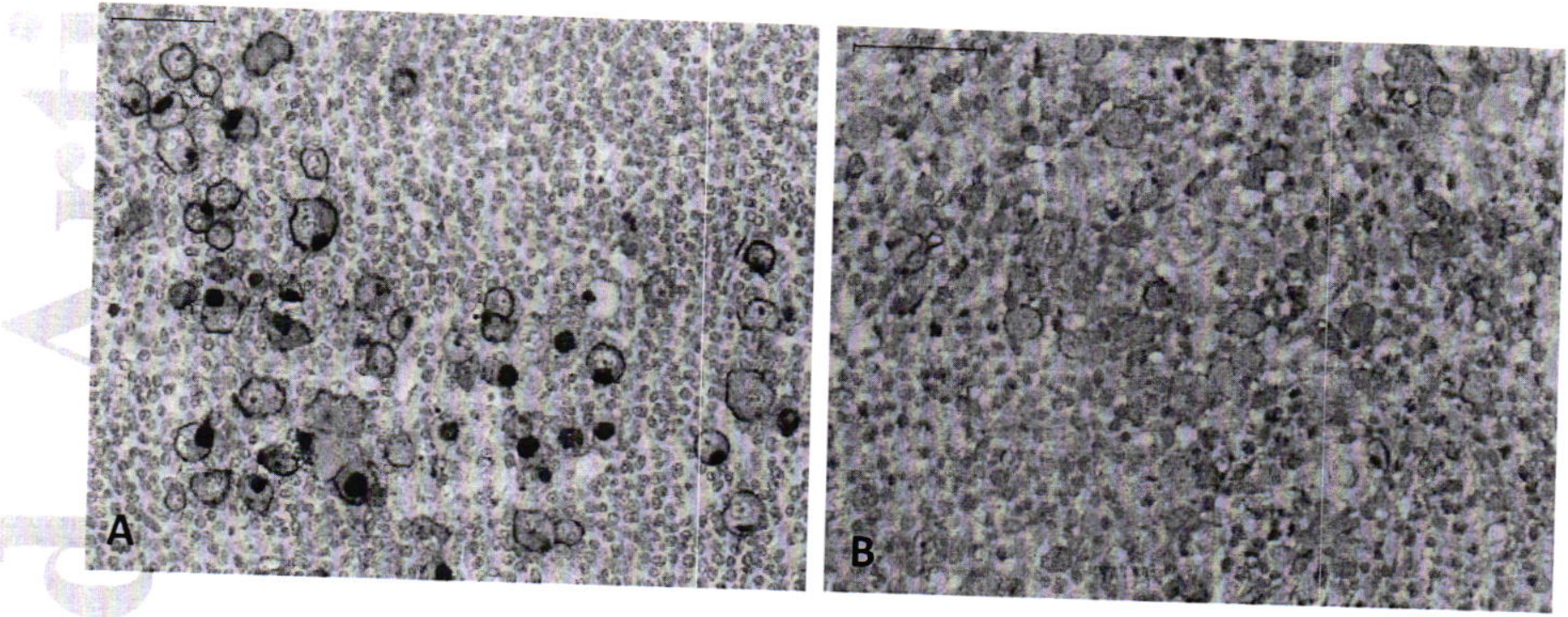

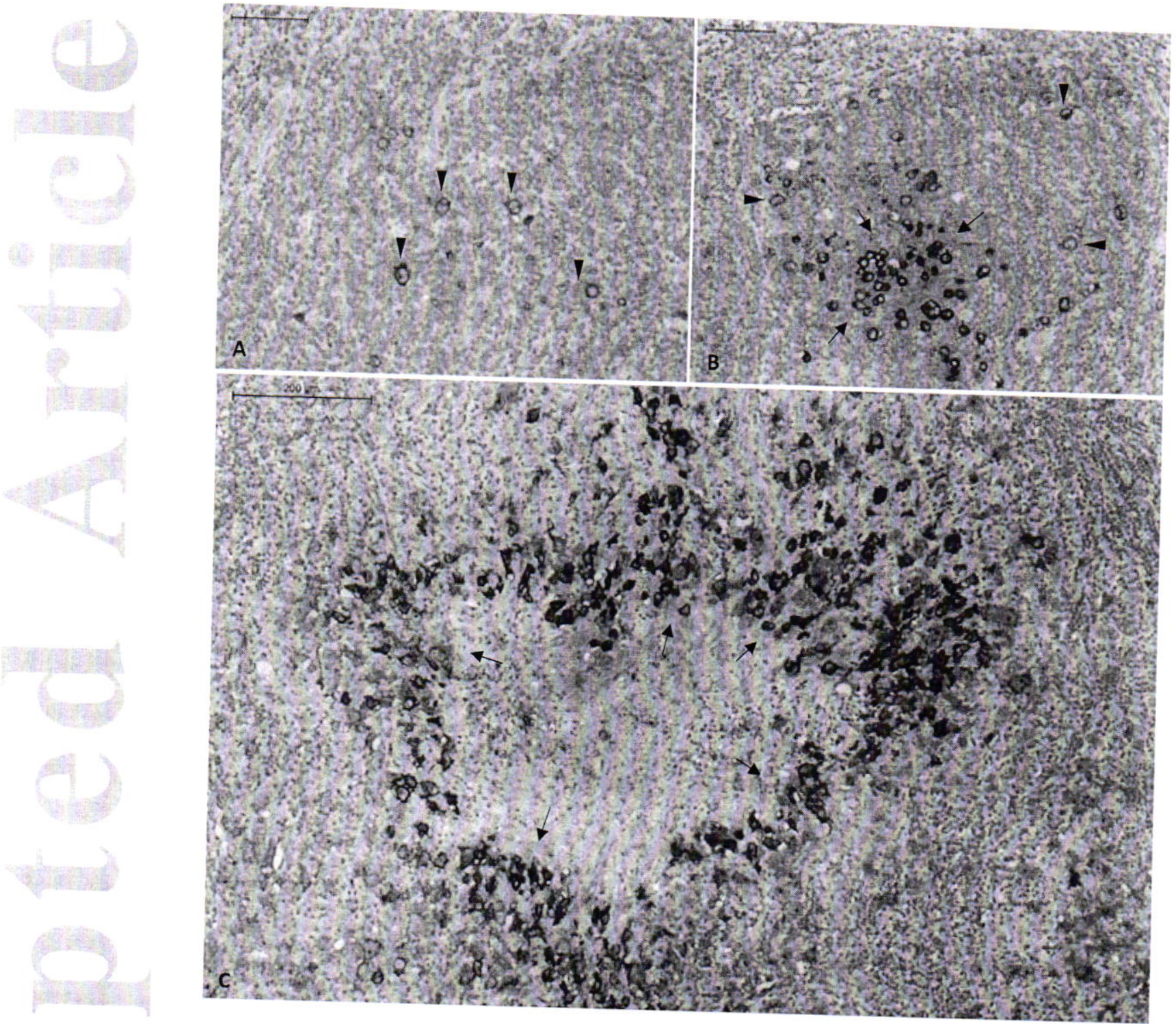

This article is protected by copyright. All rights reserved. 
Figure 3A and B

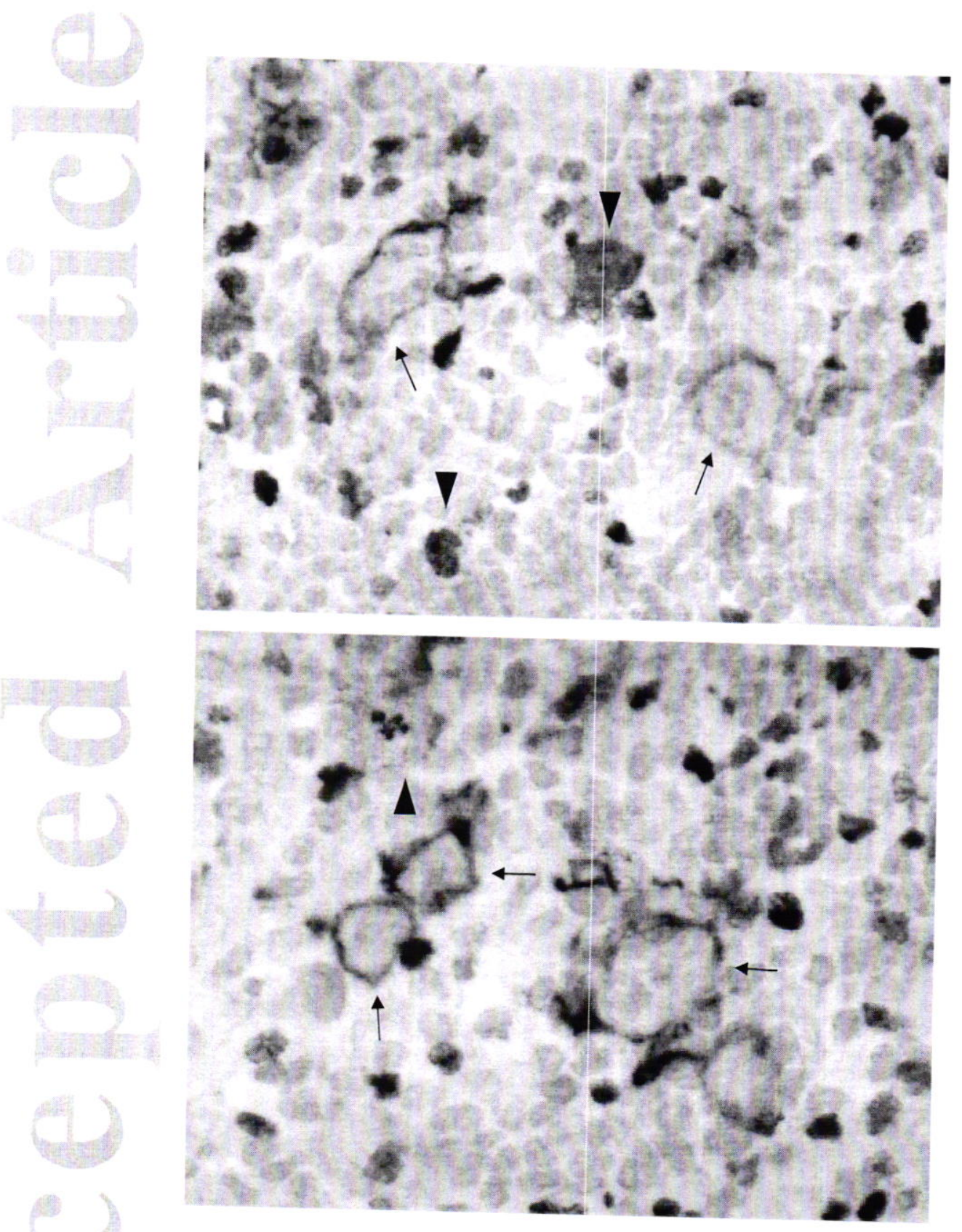


Figure 4. Ki-67 labeling index determined separately in CAIX+ and CAIX- HRS cell compartments within the same untreated $\mathrm{CHL}$ biopsy samples featuring CAIX expressing clusters and applicable for dual IHC analysis $(n=26)$. Ki67 labeling index proved to be arrest.
signty lower in CAIX+ HRS-cells $(p<0.001)$ indicating to adaptation related cell-cycle

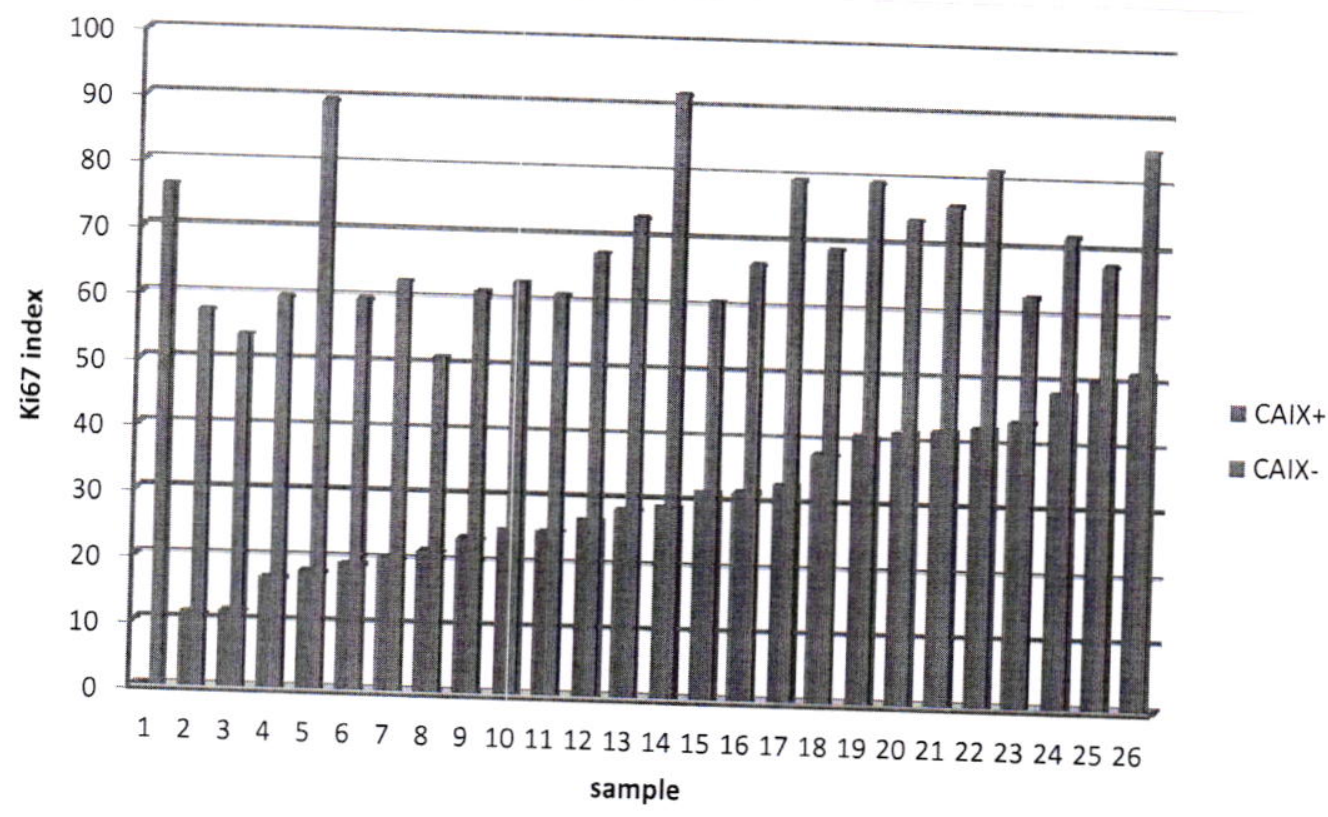


Figure 5.

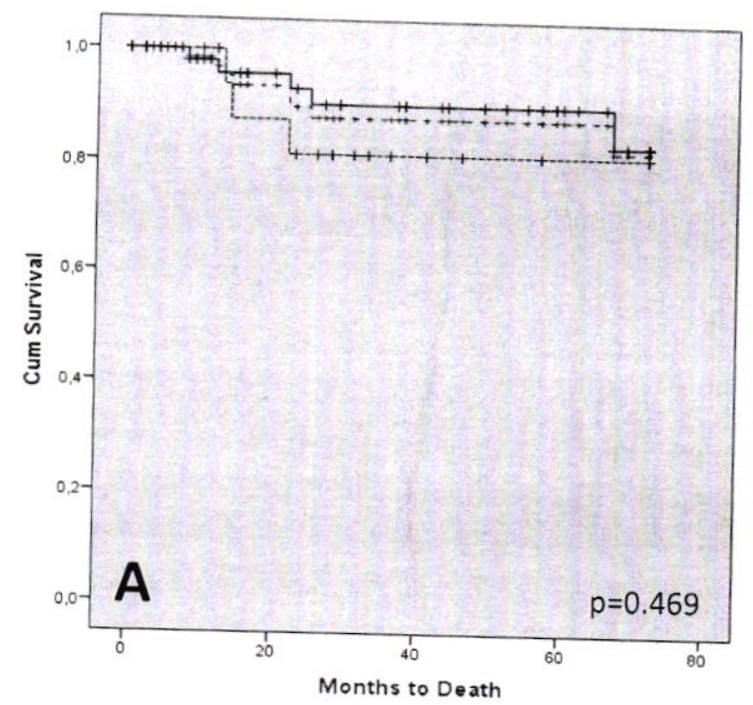

B

$p=0.146$

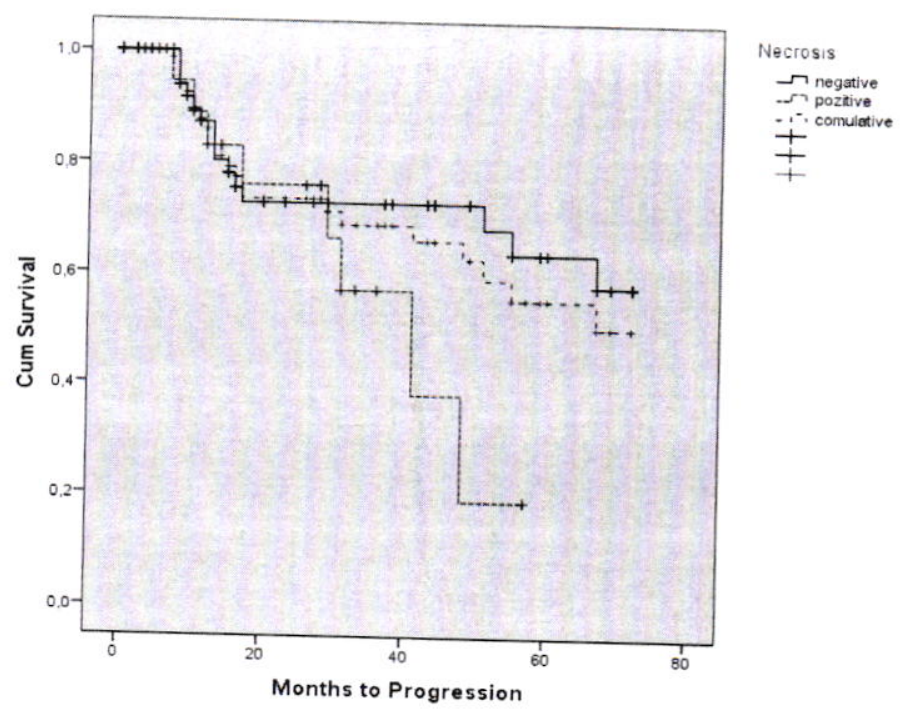




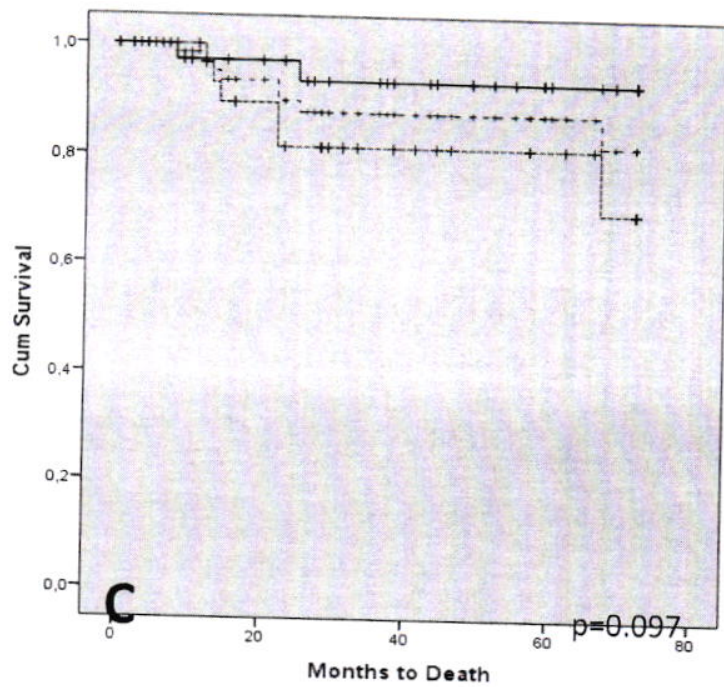

D

$p<0.001$

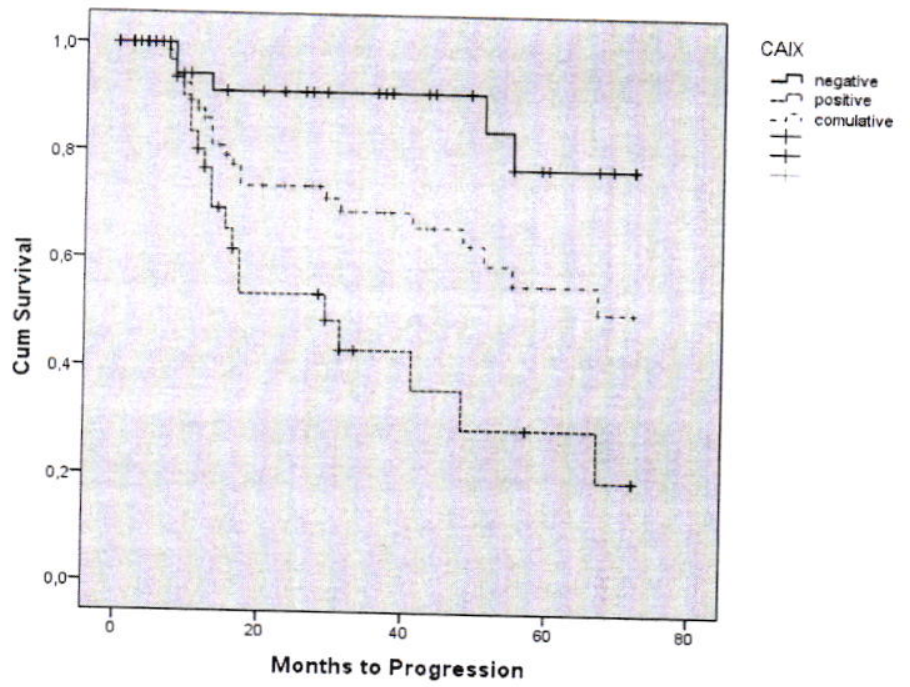




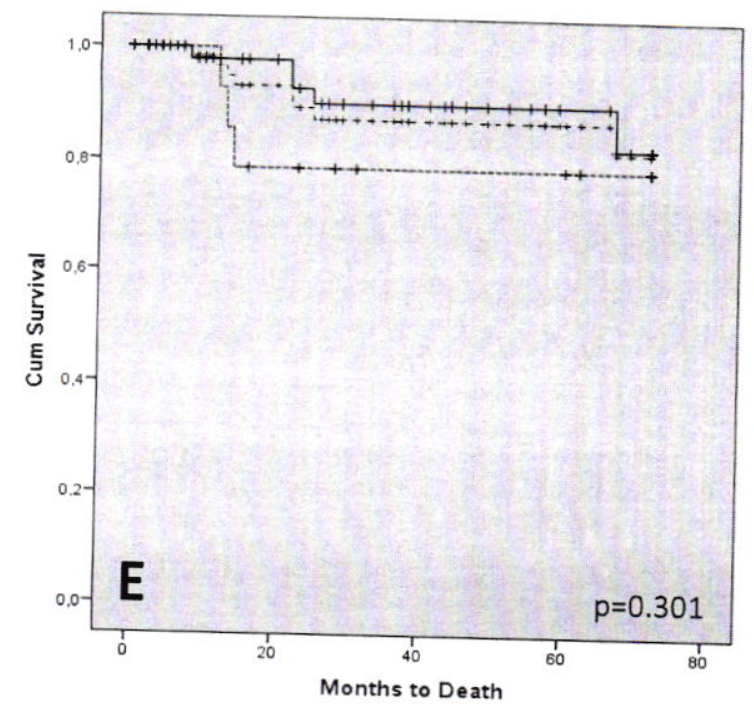

F

$p=0.524$

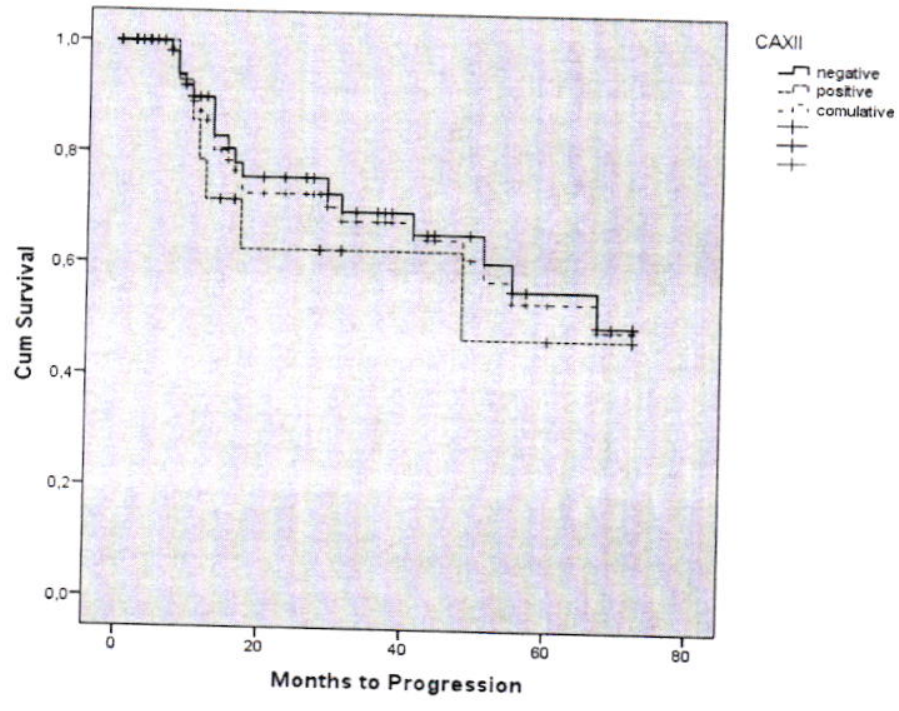

